\title{
Right ventricular free wall strain in acutely decompensated heart failure patients with ischemic and non-ischemic cardiomyopathy
}

\author{
Dino Mirić ${ }^{1}$, Ana Barac ${ }^{2}$, Vesna Čapkun ${ }^{1}$, and Darija Baković Kramarić ${ }^{3}$ \\ ${ }^{1}$ University Hospital Centre Split \\ ${ }^{2}$ MedStar Georgetown University Hospital \\ ${ }^{3}$ University Hospital Center Split Križine
}

April 6, 2021

\begin{abstract}
Aims. Right ventricular (RV) dysfunction is a predictor of adverse outcomes among patients with HF with reduced ejection fraction (HFrEF) however, differences in RV parameters in HFrEF patients with ischemic (ICM) and non-ischemic cardiomyopathies (NICM) are not well understood. We investigated echocardiographic characteristics, including RV strain, in patients with acute decompensated heart failure (ADHF) and compared patients with ICM and NICM etiology. Methods. Consecutive patients who presented with ADHF and NYHA class III-IV were prospectively enrolled if they had LVEF $<40 \%$ and history of ICM or NICM. All patients underwent clinical exam, laboratory evaluation and 2-D echocardiographic assessment of the left ventricular (LV) and RV function, LV and RV global longitudinal strain (LVGLS, RVGLS), and RV free wall strain (RVfwLS). Results. Of 84 patients, 44 had ICM and 40 NICM. The groups had similar blood pressure, NT-proBNP, and echocardiographic parameters of LV function including LVGLS. Absolute RVGLS values were lower than RVfwLS values in both groups. Patients with NICM had significantly lower RVfwLS, but not RVGLS, compared to patients with ICM (-13\% to $-17 \%$, P=0.006). Similar differences in RVfwLS were seen in patients in NYHA class III (NICM vs ICM: $-13 \%$ and -17\%, respectively, $95 \%$ CI: -8.5 to -0.5 ) and NYHA class IV (NICM vs ICM: $-13.8 \%$ and $-17 \%$, respectively, $95 \%$ CI: -6.4 to -0.59 ). Conclusion. Among patients hospitalized with ADHF, patients with nonischemic etiology compared with the patients with ICM, have worse RV dysfunction measured by RVfwLS, despite similar extent of LV impairment and the same functional limitation class.
\end{abstract}

\section{Hosted file}

Manuscript_echocardiography.odt available at https://authorea.com/users/406168/articles/ 516930-right-ventricular-free-wall-strain-in-acutely-decompensated-heart-failurepatients-with-ischemic-and-non-ischemic-cardiomyopathy 


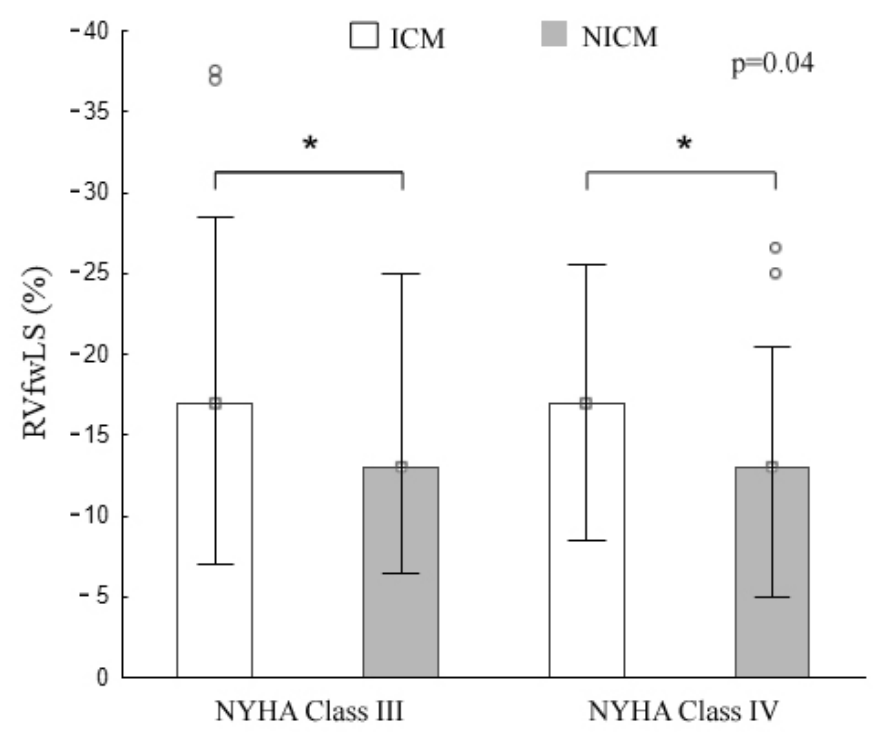

